PlastOx 2007 (2009) 255-264

(C) EDP Sciences, 2009

DOI: $10.1051 /$ ptox/2009018

\title{
Les systèmes barrières thermiques pour aubes de turbine
}

\author{
R. Mévrel \\ ONERA - Département Matériaux Métalliques et Procédés, BP. 72, 92322 Châtillon, France \\ e-mail : remy.mevrel@onera.fr
}

\begin{abstract}
Résumé. Les systèmes barrières thermiques pour aubes de turbine sont des systèmes multicouches dont la structure et la composition évoluent à haute température. Cette évolution, qui est liée à l'interaction avec l'environnement oxydant, entraîne des modifications des champs de contraintes présents dans ces systèmes et qui peuvent provoquer leur endommagement, voire leur ruine. Ce texte synthétique présente succinctement les systèmes actuellement utilisés et la problématique de l'interaction entre aspects mécaniques et environnement dans la turbine.
\end{abstract}

\section{INTRODUCTION}

Les barrières thermiques sont utilisées depuis plus de 15 ans pour protéger des aubes de turbines aéronautiques et plus récemment de turbines terrestres. Dans cet exposé on se propose de décrire brièvement les systèmes utilisés, quelques modes de dégradation importants et les perspectives d'évolution. Les barrières thermiques font l'objet de nombreuses études actuellement et dans ce texte, compte tenu du thème de l'Ecole, nous nous concentrons sur les aspects mettant en évidence l'incidence d'interactions avec l'environnement sur les propriétés mécaniques des systèmes. Pour plus de détails et de références sur ces systèmes, on se reportera à des articles de synthèse récents [1-5].

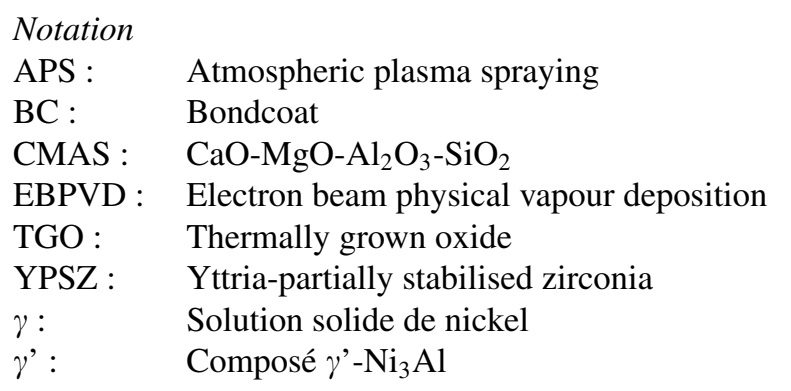

Projection plasma atmosphérique
ouche de liaison

Evaporation avec bombardement électronique Oxyde formée par oxydation Zircone yttriée partiellement stabilisée

\section{GÉNÉRALITES}

\subsection{Enjeux}

Pour améliorer les performances des turbines à gaz, plusieurs voies sont possibles, en particulier :

- Développer des matériaux capables de résister mécaniquement à des températures de plus en plus élevées. Or les superalliages à base de nickel constituant les aubes, après des progrès relatifs à leur structure (remplacement de matériaux équiaxes par des matériaux solidifiés directionnellement puis par des monograins) et à leur composition (permettant notamment des fractions volumiques très importantes de phase renforçante $\gamma^{\prime}-\mathrm{Ni}_{3} \mathrm{Al}$, sont quasiment parvenus à une asymptote quant à leur température d'utilisation [6]. D'autre part, l'utilisation de céramiques ou de siliciures n'est pas envisageable à court ou moyen terme dans ce type d'application. 


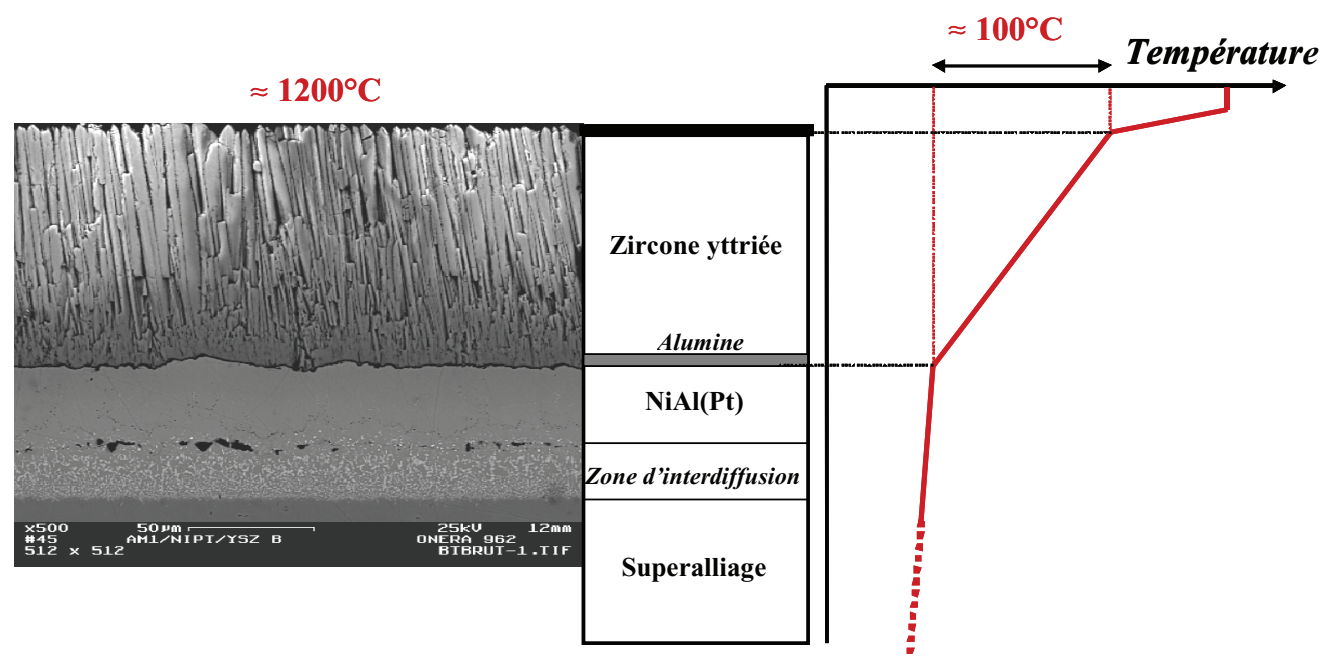

Figure 1. Schéma de principe d'une barrière thermique.

- Perfectionner la conception des composants en optimisant notamment le dessin et le fonctionnement des circuits de refroidissement de façon à conserver des températures acceptables pour le métal. Cette voie a également été largement explorée et il faut noter que la sophistication des circuits de refroidissement peut poser des problèmes de réalisation et que l'augmentation du flux d'air de refroidissement diminue le rendement du système global.

Etant donné ce contexte, la voie qui a été développée depuis les années 80 est l'application d'une barrière thermique sur les aubes mobiles et les aubes directrices. Les barrières thermiques sont appliquées sur d'autres pièces, moins sollicitées mécaniquement comme les parois de chambres de combustion et les anneaux de turbine. Dans cette présentation on se focalisera sur les problèmes posés par les barrières thermiques déposées sur aubes en raison de leur acuité.

\subsection{Barrière thermique standard}

Les barrières thermiques actuellement utilisées dans les étages chauds des turbines, qu'elles soient aéronautiques ou terrestres sont constituées d'un système multicouche (fig. 1) :

La couche céramique la plus communément utilisée dans les systèmes barrières thermiques est la zircone yttriée partiellement stabilisée $\left(\mathrm{ZrO}_{2}-6\right.$ à $8 \%$ mass. $\mathrm{Y}_{2} \mathrm{O}_{3}$ soit $4,5 \%$ mole $\mathrm{Y}_{2} \mathrm{O}_{3}$ ou encore $\mathrm{Zr}_{1-\mathrm{x}} \mathrm{Y}_{x} \mathrm{O}_{2-\mathrm{x} / 2}$ avec $\left.\mathrm{x}=0,085\right)$. Ce matériau a été sélectionné car il présente un ensemble de propriétés particulièrement intéressantes :

- Fort coefficient de dilatation pour une céramique $\left(10^{-5} \mathrm{~K}^{-1}\right.$ à rapprocher d'un coefficient typique de $14-15 \cdot 10^{-6} \mathrm{~K}^{-1}$ pour le substrat)

- Faible conductivité thermique (de l'ordre de $2 \mathrm{~W} \cdot \mathrm{m}^{-1} \mathrm{~K}^{-1}$ sous forme dense au-dessus de $800^{\circ} \mathrm{C}$ ) [7].

- Ténacité élevée (liée à la structure quadratique t' métastable [8]) pour éviter la propagation de fissures.

- Stabilité structurale à haute température (la température des gaz de combustion peut atteindre $1600^{\circ} \mathrm{C}$ et la température en surface de la couche peut atteindre $1200-1300^{\circ} \mathrm{C}$ ).

- Stabilité chimique vis-à-vis de l'environnement oxydant des gaz de combustion.

- Compatibilité avec la couche d'alumine sous-jacente.

- Compatibilité avec un procédé de dépôt comme l'EBPVD ou la projection plasma. 


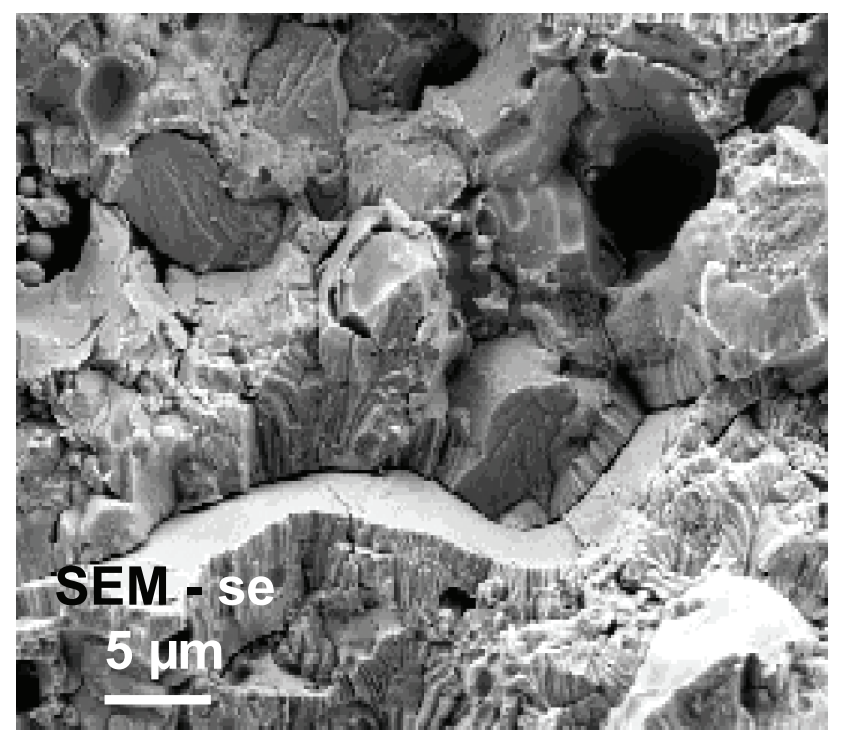

Figure 2. Détail d'un revêtement de zircone yttriée déposé par projection plasma (clinché ONERA).

Son épaisseur est en général comprise entre 150 et $300 \mu \mathrm{m}$. Elle peut être de l'ordre du mm sur les anneaux de turbine.

Dans les systèmes peu sollicités mécaniquement, elle est déposée par projection plasma, Sa structure présente une porosité lamellaire (fig. 2) qui abaisse sa conductivité thermique vers $1 \mathrm{~W} \cdot \mathrm{m}^{-1} \mathrm{~K}^{-1}$. Pour les systèmes plus sollicités, notamment les aubes mobiles de turbines aéronautiques, elle est déposée par évaporation (EBPVD), et sa structure est colonnaire, ce qui présente un avantage pour la tenue mécanique mais au détriment des propriétés thermiques (conductivité de l'ordre de $1.5 \mathrm{~W} \cdot \mathrm{m}^{-1} \mathrm{~K}^{-1}$ ).

Cette couche de zircone est déposée sur une couche de liaison métallique elle-même déposée ou formée sur le superalliage constituant la pièce. Le rôle de la couche de liaison est d'assurer une compatibilité mécanique et chimique de l'ensemble. La zircone yttriée est un très bon conducteur de l'oxygène et, même si la couche céramique n'était pas poreuse, de l'oxygène diffuserait vers la partie métallique. Il est par conséquent essentiel que cette partie métallique puisse former une couche d'oxyde stable, à croissance lente, compatible avec les parties céramique et métallique et que cet oxyde soit adhérent à la fois au métal et à la zircone yttriée. Il s'avère que l'alumine peut remplir ce rôle [9] et les couches de liaison utilisées sont des alliages aluminoformeurs, soit à base de $\beta$-NiAl( $\mathrm{Pt}$ ), soit d'alliages $\operatorname{MCrAlY}(\mathrm{M}=\mathrm{Ni}$ et/ou Co).

Les couches de liaison en alliage MCrAlY (une composition typique est $\mathrm{Ni}-18 \% \mathrm{Co}-20 \% \mathrm{Cr}$ $16,5 \% \mathrm{Al}-0,3 \% \mathrm{Y}, \%$ at.) sont surtout utilisées dans des systèmes relativement peu sollicités mécaniquement, en particulier des turbines terrestres dont les cycles de fonctionnement présentent peu de cyclages. Comme ces couches peuvent être déposées en forte épaisseur $(>100 \mu \mathrm{m})$ par projection plasma sous pression réduite ou par codépôt électrolytique, elles constituent un réservoir d'aluminium suffisant pour répondre aux besoins de longues durées de vie recherchées (plusieurs dizaines de milliers d'heures).

En revanche, pour des pièces très sollicitées mécaniquement comme les aubages de turbines aéronautiques, les couches de liaison en $\mathrm{NiAl}(\mathrm{Pt})$, plus résistantes mécaniquement, sont préférées. Pour les élaborer, un dépôt électrolytique de platine, de 4-5 $\mu \mathrm{m}$ d'épaisseur, est suivi d'une aluminisation. L'épaisseur de ces couches est de l'ordre de 40 à $50 \mu \mathrm{m}$ et une zone d'interdiffusion de l'ordre de $30 \mu \mathrm{m}$ affecte le superalliage en contact. A l'état brut d'élaboration, la couche de liaison contient 45 à $50 \%$ at. $\mathrm{Al}$ et de l'ordre de $10 \%$ at. Pt. 


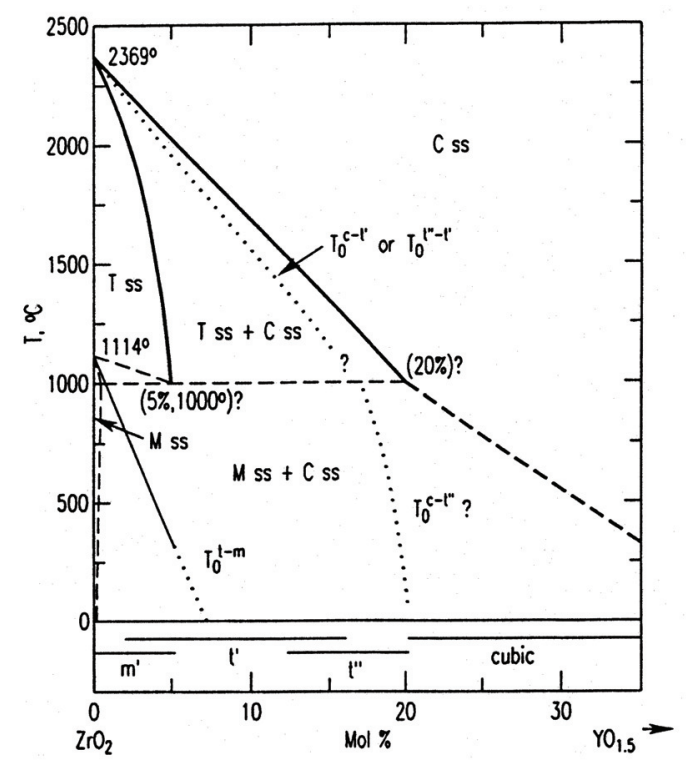

Figure 3. Diagrammen de plase $\mathrm{ZrO}_{2}-\mathrm{YO}_{1.5}$ [14].

Le superalliage est un alliage à base de nickel, constitué d'une matrice de structure $\gamma$-Ni (solution solide) cfc renforcée par une précipitation cohérente $\gamma^{\prime}-\mathrm{Ni}_{3} \mathrm{Al}$. Il est important de noter que des éléments du superalliage peuvent avoir une influence sur le comportement en oxydation du système : favorable pour des éléments comme Hf, $\mathrm{Y}, \mathrm{Zr}$ ou les terres rares, néfaste pour le soufre. Ainsi, une teneur en soufre inférieure à $0,2 \mathrm{ppm}$ (masse) est recommandée pour éviter une dégradation de l'adhérence de la barrière. Cet effet du soufre est discuté en particulier dans [10-12].

A titre d'ordre de grandeur, pour les systèmes actuels, le gradient de température dans la couche céramique peut être de $1 \mathrm{~K} / \mu \mathrm{m}$, la température maximale en surface de la couche de zircone yttriée peut atteindre localement $1200-1250^{\circ} \mathrm{C}$ et la température du métal ne doit pas dépasser en principe $1100{ }^{\circ} \mathrm{C}$. Il faut remarquer que les températures sur une aube ne sont pas constantes dans le temps ni dans l'espace.

\section{LE SYSTÈME BARRIÈRE THERMIQUE : UN SYSTÈME QUI ÉVOLUE À HAUTE TEMPÉRATURE}

En fonctionnement à haute température, le système multicouche constituant une barrière thermique évolue et cette évolution, de composition et de structure, peut modifier les propriétés mécaniques de chaque couche et celles du système dans son ensemble.

\subsection{La couche céramique}

La zircone yttriée partiellement stabilisée est métastable et la phase t' qui la constitue peut se transformer, en pratique au-dessus de $1200^{\circ} \mathrm{C}$, en un mélange de phases quadratique et cubique d'équilibre (fig. 3), avec pour conséquence une perte possible de ténacité ou une transformation néfaste de phase quadratique en monoclinique au refroidissement (voir par exemple [13]).

D'autre part, à haute température, l'architecture poreuse de la couche céramique peut évoluer, avec des phénomènes de frittage entraînant une élévation de la conductivité thermique [15] et une augmentation de sa rigidité [16]. 


\subsection{La couche d'alumine}

La couche d'alumine interfaciale s'épaissit conformément aux cinétiques typiques relatives à la croissance d' $\alpha-\mathrm{Al}_{2} \mathrm{O}_{3}$, après une éventuelle période d'oxydation transitoire. Il faut noter qu'une oxydation survient déjà durant du préchauffage de la pièce, avant le dépôt par évaporation de la zircone yttriée. Lors de cette préoxydation, des alumines de transition sont susceptibles de se former. Elles se transformeront ensuite en $\alpha-\mathrm{Al}_{2} \mathrm{O}_{3}$ stable à haute température. Il est parfois possible d'observer la mémoire de ces alumines de transition sous la forme d'une zone mixte à l'interface zircone/alumine. Il faut aussi noter que, comme ces alumines de transition croissent de façon prédominante par diffusion cationique, des cavités peuvent se former à l'interface alumine/métal et constituer des amorces de défauts. La croissance d' $\alpha-\mathrm{Al}_{2} \mathrm{O}_{3}$ s'accompagne de contraintes de croissance (en compression), vraisemblablement liées à la formation de nouvel oxyde au sein même de la couche d'oxyde [17].

\subsection{Couche de liaison}

La composition de la couche de liaison évolue sous l'influence de deux phénomènes :

- Son oxydation, avec corrélativement une diminution progressive de sa teneur en aluminium

- L'interdiffusion avec le substrat [18].

En conséquence, la structure de la couche de liaison peut évoluer, avec, dans le cas de couches $\beta$-NiAl(Pt) l'apparition de phase $\gamma$ ' voire $\gamma$, transformations qui peuvent s'accompagner de variations de volume et celles de ses propriétés mécaniques.

D'un autre côté, des éléments du substrat peuvent diffuser dans la couche de liaison et modifier les propriétés mécaniques de cette couche ou affecter les phénomènes d'oxydation.

\subsection{Superalliage}

Les phénomènes d'interdiffusion peuvent également modifier la composition de la zone superficielle du superalliage. A noter en particulier que pour les superalliages les plus récents (dits de 4 ème génération), une zone de réaction secondaire peut se développer par interaction avec la couche de liaison [19]. En conséquence, la section utile du superalliage peut s'en trouver réduite, ce qui peut être néfaste à l'endroit de sections minces.

\section{MODES DE DÉGRADATION. MÉCANISMES}

\subsection{Introduction}

En fonctionnement, les systèmes barrières thermiques sont soumis à l'agression du milieu environnant: oxydation par les gaz de combustion (qui peuvent contenir outre de l'oxygène, de la vapeur d'eau), impacts de particules, dépôts d'oxydes. Ces interactions peuvent être à l'origine de contraintes provoquant des déformations affectant l'intégrité du système.

S'y ajoutent des contraintes d'origine thermique (liées aux différences de coefficients de dilatation) ou externes (contraintes liées à la force centrifuge, aux sollicitations de fatigue, mécaniques, thermiques, mécanothermiques). La situation est particulièrement complexe et on va illustrer de façon très simplifiée deux modes de dégradation mettant en jeu le couplage oxydation/mécanique.

\subsection{Cyclage thermique en atmosphère oxydante}

\subsubsection{Oxydation}

L'oxygène diffuse rapidement au travers de la couche céramique car la zircone yttriée présente une concentration élevée de lacunes d'oxygène - pour une composition typique $\mathrm{Zr}_{1-\mathrm{x}} \mathrm{Y}_{\mathrm{x}} \mathrm{O}_{2-\mathrm{x} / 2}$, la 


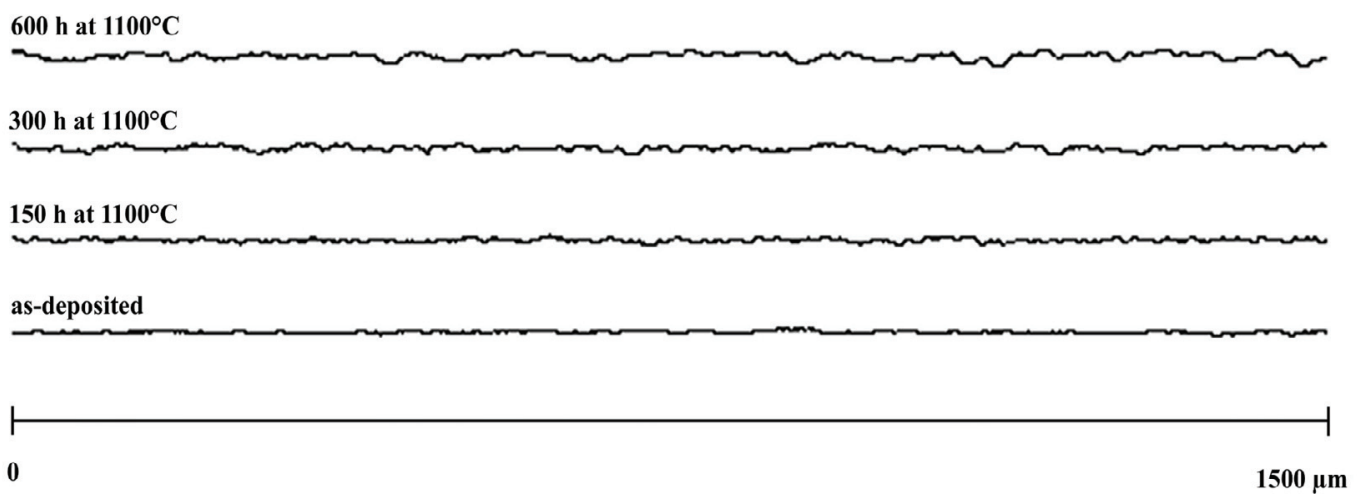

Figure 4. Évolution de la rugosité interfaciale dans une barrière thermique EBPVD après divers traitements isothermes à $1100^{\circ} \mathrm{C}[20]$. Les valeurs de rugosité arithmétique Ra correspondantes sont de $1 \mu \mathrm{m}, 1,5 \mu \mathrm{m}, 2,1 \mu \mathrm{m}$ et $2,75 \mu \mathrm{m}$.

proportion de lacunes sur les sites oxygène est $\mathrm{x} / 4$, soit $2 \%$ dans une YPSZ - et c'est d'ailleurs cette propriété qui est à l'origine de sa faible conductivité thermique.

Cet oxygène participe à la croissance de la couche d'alumine qui se développe à l'interface entre couche de liaison et couche céramique. Cette oxydation s'accompagne de contraintes de croissance (en compression à long terme), sous l'influence desquelles la couche d'alumine peut se déformer, ce qui va contribuer à augmenter la rugosité de l'interface couche de liaison/alumine (fig. 4).

\subsubsection{Effet du cyclage thermique}

Les différentes couches qui constituent le système barrière thermique ont des coefficients de dilatation différents. Le cyclage thermique du système constitué par le superalliage et la couche de liaison aboutit à une accumulation de déformation plastique dans la couche de liaison et qui se manifeste par une augmentation de la rugosité (« rumpling » ou « ratcheting »). Cet effet peut se cumuler avec la déformation induite par la croissance de la couche d'alumine.

A ceci, et cela dépend de la nature de la couche de liaison et des conditions de fonctionnement, peut s'ajouter l'effet de variations de volume associées à la transformation martensitique de la couche de liaison. En effet, pour les faibles teneurs en aluminium, la phase $\beta-\mathrm{NiAl}(\mathrm{Pt})$ peut se transformer en martensite au refroidissement avec une variation de volume estimée à environ $3 \%$ [21].

En conséquence, à température ambiante, la couche d'alumine est sujette à de fortes contraintes résiduelles. Ces contraintes peuvent être déterminées par piezospectroscopie [22, 23] et les valeurs obtenues sont de l'ordre de 3 à $5 \mathrm{GPa}$ en compression.

Etant donné que l'interface céramique/métal est rugueuse, ces contraintes de compression se traduisent par des contraintes en tension aux sommets et dans les vallées de la couche d'alumine (respectivement à l'interface alumine/métal et à l'interface zircone/alumine) [24]. Selon l'adhérence des différentes couches, une décohésion peut survenir à l'une ou l'autre des interfaces, avec formation de cavités et de fissures comme l'illustre la fig. 5.

Progressivement, ces fissures vont croître, coalescer et se propager, entraînant notamment une diminution de l'énergie d'adhérence (fig. 6) et, in fine, l'énergie emmagasinée dans le système (d'autant plus grande que la couche d'alumine est épaisse), peut conduire au décollement de la couche céramique (fig. 7) lors d'un refroidissement.

La description mécanique du phénomène d'évolution de rugosité (ratcheting ou rumpling) à l'origine de ce mode de dégradation a été abordée par plusieurs groupes, notamment par Balint et al. [26] au moyen d'une approche semi-analytique et d'autres auteurs [27-29] au moyen de calculs par éléments 

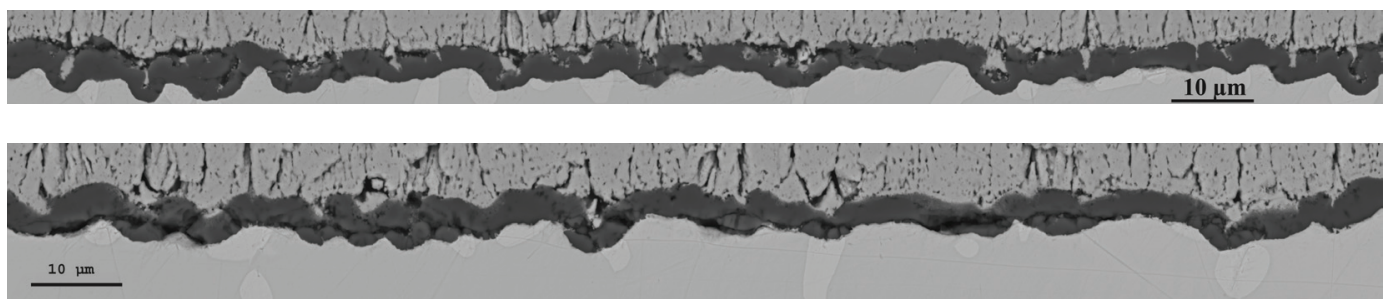

Figure 5. Endommagements dans la zone interfaciale d'une barrière thermique (clichés ONERA).

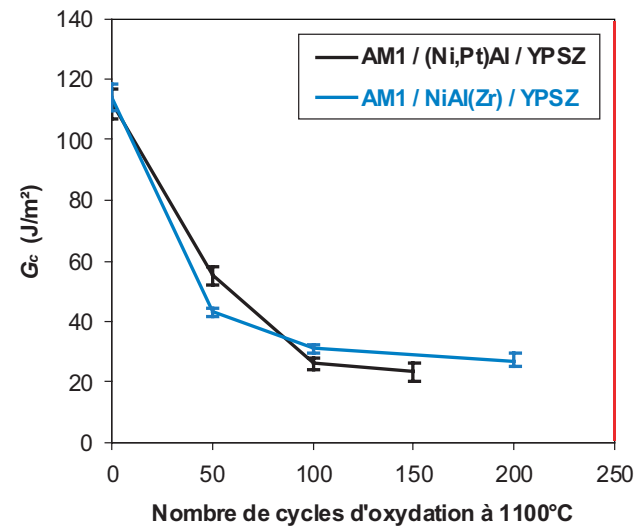

Figure 6. Évolution de l'energie d'adhésion en fonction du nombre de cycles à $1100{ }^{\circ} \mathrm{C}$ pour deux sysémes barriéres thermiques [25].

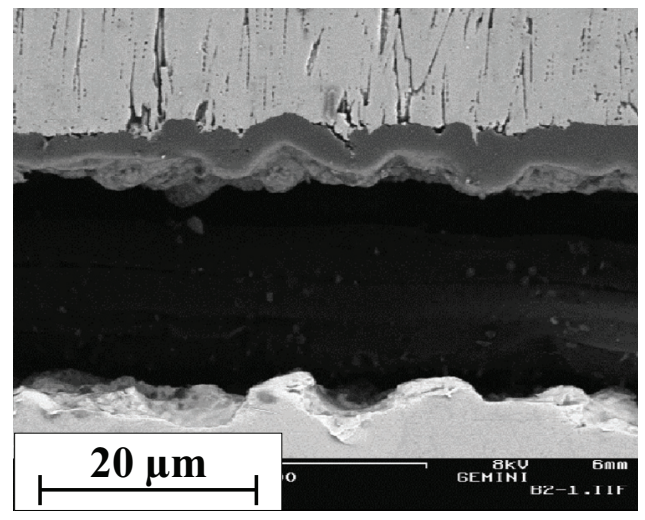

Figure 7. Décollement de barriére thermique aprés cyclage à $1100^{\circ} \mathrm{C}$ (cliché ONERA).

finis. Une difficulté reste l'introduction dans ces calculs de lois de comportement pertinentes, notamment pour la couche de liaison. Il est à souligner que la détermination de ces lois (élasto-viscoplastiques) fait l'objet de travaux actuellement, avec des essais sur microéprouvettes [30], sur systèmes composites [31] et par microindentation instrumentée à haute température [32].

La fig. 8 montre un exemple de carte obtenue à partir de calculs selon l'approche de Balint et Hutchinson [33]. Il faut lire cette carte de la façon suivante. Pour une contrainte de croissance de l'oxyde donnée, et pour une différence donnée de coefficients de dilatation entre couche de liaison et superalliage, le phénomène de « ratcheting » peut se produire si la résistance en fluage de la couche de liaison est inférieure à une certaine valeur. En d'autres termes, ce schéma illustre que pour éviter ce phénomène de « ratcheting », il faut :

- une couche de liaison résistante en fluage,

- une faible différence de dilatation entre couche de liaison et superalliage (y compris les différences associées aux changements de phase)

- des contraintes de croissance de l'alumine faibles.

\subsection{Dégradation par interaction avec dépôts de CMAS}

En fonctionnement, le moteur ingère diverses particules (sable, poussières, débris). De plus, des particules contenant du fer, du titane notamment peuvent se détacher de pièces du moteur situées en amont. Toutes ces particules peuvent se déposer à la surface de la couche céramique et il n'est pas inhabituel d'observer de tels dépôts (fig. 9) sur des aubes après fonctionnement dans un moteur [35]. 


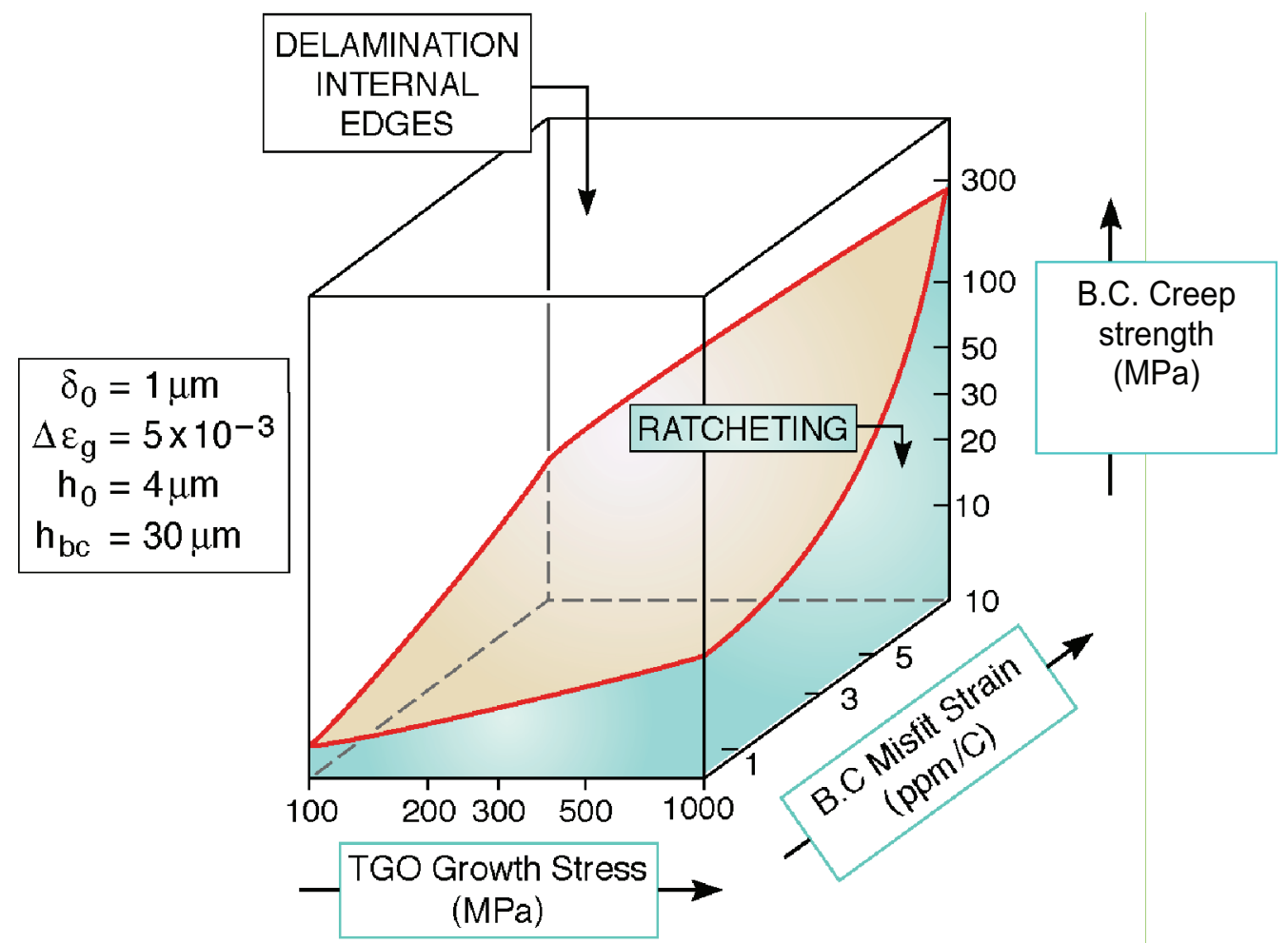

Figure 8. Carte des modes d'endommagement de barrières thermiques (d'après [34]; $\delta_{0}$ est l'épaisseur de la couche d'alumine, $\Delta \varepsilon_{\mathrm{g}}$ est la déformation associée à la croissance de l'alumine, $\mathrm{h}_{0}$ est l'amplitude des ondulations et $\mathrm{h}_{\mathrm{bc}}$ est l'épaisseur de la couche de liaison).

De plus il a été constaté que certains dépôts peuvent s'infiltrer dans les espaces intercolonnaires et que parfois des réactions avaient pu avoir lieu avec la couche céramique.

Ces dépôts sont constitués d'oxydes contenant les éléments $\mathrm{Ca}, \mathrm{Mg}, \mathrm{Al}$ et $\mathrm{Si}$ (d'où leur appellation CMAS). Il se trouve que ces dépôts peuvent avoir des points de fusion proches de la température en surface de la couche céramique. Leur faible viscosité à ces températures explique le phénomène d'infiltration.

Le phénomène peut avoir deux conséquences : rigidifier la partie céramique infiltrée et entraîner une fissuration lors de cyclages thermiques (fig. 10). Ce phénomène a été décrit par une équipe de l'UCSB et une approche mécanique en a été proposée récemment [36, 37].

D'autre part, une interaction chimique est possible entre ces silicates et la zircone yttriée avec phénomènes de dissolution/précipitation, ce qui risque d'entraîner la ruine du système.

Il est important de noter que ce phénomène est d'autant plus aigu que la température est élevée, ce qui d'ailleurs pourrait mettre un frein à la recherche de températures toujours plus élevées dans ces systèmes.

\section{CONCLUSION}

Les barrières thermiques sont des systèmes utilisés en production depuis plus d'une quinzaine d'années sur des pièces critiques de turbine comme les aubes mobiles. Des expériences de laboratoire, sur banc de combustion, sur moteur de banc d'essai puis les retours d'expérience après fonctionnement en vol, 


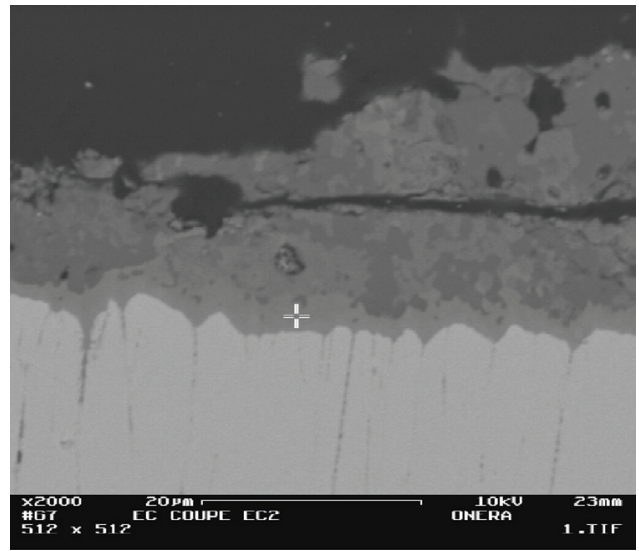

Figure 9. Dépôt de CMAS à la surface d'une barriére thermique apré fonctionement dans un moteur ; noter 1 'infiltration dans les espaces intercolonnaires (cliché ONERA).

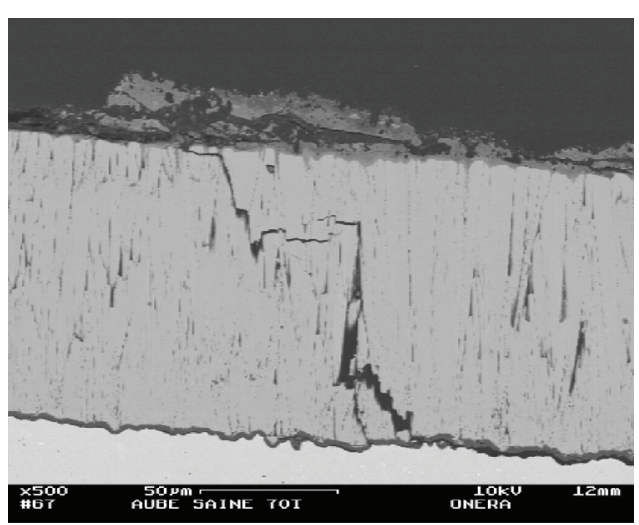

Figure 10. Fissuration dans une barriére thermique sous un dépôt de CMAS (cliché ONERA).

ont permis d'en améliorer les performances. Il n'en reste pas moins qu'une marge de progression existe pour réduire la dispersion des propriétés et mieux exploiter les potentialités de ces systèmes.

On commence à disposer de bonnes descriptions des mécanismes de dégradation. Ces mécanismes peuvent être complexes car mettant en jeu des phénomènes couplés de diffusion, d'oxydation et de déformation. Plusieurs groupes [38] développent des descriptions mécaniques de ces systèmes prenant en compte l'ensemble de ces aspects, l'objectif étant, à terme, de développer des modèles de prévisions de durée de vie fondés sur une description physique des phénomènes.

\section{Remerciements}

Ce texte a été rédigé à partir de travaux effectués à l'ONERA, notamment par M. Poulain, O. Lavigne, M.-H. Vidal-Sétif et dans le cadre des thèses de A. Azzopardi, S. Margueron, M. Caliez, Y. Cadoret, P.-Y. Théry. Ces travaux ont bénéficié des compétences métallographiques et analytiques de Catherine Rio dont les clichés illustrent ce texte. Je remercie également A. Malié (Snecma-Services) et F. Bourlier (Snecma-Moteurs) pour des discussions fructueuses.

\section{Références}

[1] Evans A.G., Mumm D.R., Hutchinson J.W., Meier G.H., Pettit F.S., Progress in Materials Science 46, 505 (2001).

[2] Padture N.P., Gell M., Jordan E.H., Science 296, 280 (2002).

[3] Clarke D.R., Levi C.G., Annual Rev. Materials Research 33, 383 (2003).

[4] Levi C.G., Current Opinion in Solid State and Materials Science 8, 77 (2004).

[5] Gleeson B., Journal of Propulsion and Power 22, 375 (2006).

[6] Reed R.C., Superalloys. Fundamentals and applications. Cambridge University Press (2006).

[7] Mévrel R., Laizet J.-C., Azzopardi A., Leclercq B., Poulain M., Lavigne O., Demange D., J. European Ceramic Society 24 (2004) 3081-9.

[8] Mercer C., Williams J.R., Clarke D.R., Evans A.G., Proc. Royal Soc. A 463, 1393 (2007)

[9] Monceau D., ce volume.

[10] Rouzou I., Molins R., Rémy L., Jomard F., Materials Science Forum 461-464, 101 (2004). 
[11] Molins R., Rouzou I., Hou P., Oxid. Metals 65, 1573 (2006).

[12] Cadoret Y., Effet du platine et du soufre sur l'oxydation des revêtements protecteurs $\beta$-NiAl à haute température. Applications aux barrières thermiques. Thèse de l'université de Paris VI (2005) préparée à l'ONERA et à l'ENSCP.

[13] Azzopardi A., Mévrel R., Saint-Ramond B., Olson E., Stiller K., Surf. and Coat. Technology 177-178, 131 (2004).

[14] Yashima M., Kakihana M., Yoshimura M., Solid State Ionics 86-88, 1131 (1996). Voir aussi: Fabrichnaya O. and Aldinger F., Zeitschrift für Metallkunde 95, 27 (2004).

[15] Poulain M., Etude de la conductivité thermique de revêtements céramiques à base de zircone. Relation avec la composition, la microstructure et la morphologie. Thèse de l'Université de ParisSud (1999) préparée à l'ONERA.

[16] Azzopardi A. Evolution microstructurale à haute température de barrières thermiques déposées par évaporation. Influence sur la conductivité thermique et le module d'élasticité. Thèse de l'Université de Paris VI, (2003) préparée à l'ONERA.

[17] Clarke D., Acta Mater. 51, 1393 (2003).

[18] Larnicol M., Rio C., Renollet Y., Pioche Y., Mévrel R., J. Phys. IV, 10, Pr4, 161-166 (Mars 2000).

[19] Lavigne O., Ramusat C., Drawin S., Caron P., Boivin D., and Pouchou J.-L., Relationships Between Microstructural Instabilities and Mechanical Behaviour in New Generation NickelBased Single Crystal Superalloys, in Superalloys 2004, eds. Green K.A., Pollock T.M., Harada H. (Warrendale, PA: TMS, 2004), pp. 667-675.

[20] Poulain M., Dorvaux J.-M., Mévrel R., Rio C., Ritti M.-H., Evolution of the alumina layer / bondcoat interface in oxidised EBPVD TBCs in Surface Modification technologies XVIII, Ed. T.S. Sudarshan, M. Jeandin, J.J. Stiglich, MANEY - ASM International, 405-409 (2006).

[21] Karlsson A.M., Trans. ASME 125, 346 (oct. 2003)

[22] Christiensen R.J., Lipkin D.M., Clarke D.R., Murphy K., Appl. Phys. Lett. 69, 3754 (1996).

[23] Margueron S., Evaluation de la méthode de piezospectroscopie en vue d'un contrôle nondestructif de barrières thermiques EB-PVD. Thèse de doctorat du CNAM (2003) préparée à l'ONERA.

[24] Clarke D.R., Pompe W., Acta Mater. 471749 (1999)

[25] Théry P.-Y.,Adhérence de barrières thermiques pour aube de turbine avec couches de liaison $\beta$-(Ni, Pt)Al ou $\beta$-NiAl(Zr). Thèse de l'université Joseph Fourier (2007) préparée à l'ONERA.

[26] Balint D.S., Xu T., Hutchinson J.W., Evans A.G., Acta Mater. 54, 1815 (2006).

[27] Caliez M., Feyel F., Kruch S., Chaboche J.-L., Surf. Coat. Technol. 157, 103 (2002).

[28] Busso E., Wright L., Evans H.E., McCartney L.N., Saunders S.R.J., Osgerby S., Nunn J., Acta Mater. 55, 1491 (2007).

[29] Bednarz P., Finite element simulation of stress evolution of thermal barrier coating systems. Schriften des Forschungzentrums Jülich, Vol. 60 (2007).

[30] Pan D., Chen M.W., Wright P.K., Hemker K.J., Acta Mater. 51, 2205 (2003).

[31] Taylor M.P., Ebvans H.E., Busso E.P., Qian Z.Q., Acta Mater. 54, 3241 (2006).

[32] Passilly B., Kanouté P., Leroy F.-H., Mévrel R., Phil. Mag. 86, 5739 (2006).

[33] Balint D.S., Hutchinson J.W., J. Mech. Phys. Solids 53, 949 (2005).

[34] Evans A.G., Clarke D.R., Levi C.G., Hutchinson J.W., Gleeson B., Thermal barrier systems for aero-engines, in TBC Workshop, UCSB (jan 2007).

[35] Vidal-Sétif M.-H., Rio C., Mévrel R., Etude pilote retour d'expérience barrière thermique. Expertise ONERA. ONERA RT 1/08552 DMMP (2003).

[36] Mercer C., Faulhaber S., Evans A.G., Darolia R., Acta Mater. 53, 1029 (2005).

[37] Krämer S., Yang J., Levi C., Johnson C.A., J. Amer. Ceram. Soc. 89, 3167 (2006).

[38] Par exemple : University of California Santa Barbara (UCSB), Harvard, Johns Hopkins University, Imperial College, Ecole des Mines de Paris, ONERA, Centre de Jülich, etc. 